\title{
What lies behind chemotherapy-induced ovarian toxicity?
}

\author{
Irit Ben-Aharon ${ }^{1,3}$ and Ruth Shalgi ${ }^{2,3}$ \\ ${ }^{1}$ Institute of Oncology, Davidoff Center, Rabin Medical Center, Petah-Tiqva, Israel, ${ }^{2}$ Department of Cell and \\ Developmental Biology and ${ }^{3}$ Sackler Faculty of Medicine, Tel Aviv University, Ramat-Aviv, Tel Aviv 69978, Israel
}

Correspondence should be addressed to R Shalgi; Email: shalgir@post.tau.ac.il

\begin{abstract}
Seminal advances in anticancer therapy as well as supportive care strategies have led to improved survival rates, posing an emphasis on preserving an optimum quality of life after cancer treatment. This recognition has paved the way to an increasing research of long-term side effects, both clinical and preclinical and to an ongoing design of a supportive care system to evaluate and treat long-term adverse effects of anticancer treatments, including the impact on fertility. As with many adverse effects induced by anticancer treatments, the literature comprised mostly clinical data with regard to chemotherapy-induced gonadotoxicity, while understanding of the biological mechanism is lagging. The impact of anticancer treatments on female fertility depends on the women's age at the time of treatment, the chemotherapy protocol, the duration, and total cumulative dose administered. Several suggested mechanisms that underlie chemotherapy-induced gonadotoxicity have been described. This review illustrates the clinical evidence, as well as its supportive preclinical studies, while proceeding from the 'bedside to the bench work' and provides an insight to what lies behind chemotherapyinduced gonadotoxicity.

Reproduction (2012) $\mathbf{1 4 4} 153-163$
\end{abstract}

\section{Introduction}

Novel approaches in early detection and effective management strategies have led to increased rates of cancer survivors throughout the past three decades. In 2008, 1 435000 people were diagnosed in the US with cancer, $4 \%$ ( $\sim 57500$ cases) of them were under the age of 35. The most frequent malignancies in people under 40 are breast cancer, cervical cancer, sarcoma, non-Hodgkin's lymphoma, leukemia, and melanoma (Jemal et al. 2008). Out of an estimated 2.4 million breast cancer survivors in the US, $10 \%$ are of childbearing age. Seminal advances in anticancer therapy as well as supportive care strategies result in improved survival rates, thus yielding an ancillary focus on preservation of an optimum quality of life after cancer treatment. This recognition has paved the way to an increasing research of long-term side effects, both clinical and preclinical and to an ongoing design of a supportive care system to evaluate and treat long-term adverse effects of cancer treatments, including the impact on fertility.

As a long-term adverse outcome of anticancer treatments, the literature comprised mostly clinical data, while understanding of the biological mechanism is lagging. This review illustrates the clinical evidence as well as its supportive preclinical studies, while going from the 'bedside to the bench work' and providing an insight into what lies behind gonadotoxicity.

\section{The impact of anticancer treatments on fertility: clinical evidence}

Several studies have assessed reproductive success after cancer itself and following anticancer treatments. The Childhood Cancer Survivor Study (CCSS) found that female survivors and partners of male survivors were substantially less likely to have live births compared with their siblings. Recently published, the updated CCSS demonstrated that $\sim 30 \%$ of male childhood cancer survivors had permanent infertility (Sklar et al. 2006). Men aged 15-44 years, who received either testicular radiation at a dose of more than $7.5 \mathrm{~Gy}$, or who were treated with procarbazine or cyclophosphamide were less likely to achieve a pregnancy. Men diagnosed in early childhood were more likely to father a pregnancy than those diagnosed in adolescence (Green et al. 2010). A study published in 2008 found an increase in the use of assisted reproductive technologies (ART) with both male and female cancer patients, and a significant decrease in first-time parental probability in female patients compared with the general population (Magelssen et al. 2008). Two Scandinavian cohort studies compared $\sim 25000$ childhood, adolescent, and young adult survivors with their siblings. These studies found that the relative probability of a cancer survivor having a child was reduced by about $50 \%$ for women and about $30-57 \%$ for men (Madanat et al. 2008, Schover 2008). 
There are several obstacles to drawing clear conclusions regarding the gonadotoxic potential of various anticancer treatments. The effects of chemotherapy and radiation therapy on fertility depend on the patient's age, chemotherapeutic regimens, dose and duration, the size and location of the radiation field, type of cancer (mainly in male patients), and pretreatment fertility status of the patient. The major drawback in assessing the rate of infertility is the use of inaccurate parameters like amenorrhea as gonadal outcomes in most of the studies, as this is only a surrogate marker for infertility, while many women with regular menstrual cycles are not fertile.

Hormonal measurements to appraise the ovarian reserve have been evaluated in recent studies. FSH and estradiol $\left(E_{2}\right)$ measured on day 3 of the menstrual cycle reflect the population of maturing follicles and are indirectly associated with ovarian reserve. Inhibin- $\beta$, secreted by the granulosa cells lining the follicles, is directly associated with the loss of oocytes; however, the assay is not very reliable. Anti-Müllerian hormone $(\mathrm{AMH})$, secreted by the granulosa cells of follicles, varies relatively little through the menstrual cycle and can thus be measured at any time. AMH levels appear to be more sensitive predictors of the ovarian reserve loss; they become abnormal earlier than follicular FSH (Lobo 2005). Ultrasound imaging of the ovaries early in the menstrual cycle (days 2-4) with a count of antral follicles (measuring 2-10 mm in both ovaries combined) is also a reliable measurement of ovarian reserve. Nevertheless, large, prospective studies are warranted to confirm that these are indeed reliable markers for evaluating chemotherapy-induced ovarian failure.

\section{The impact of anticancer treatments on female fertility}

The impact of anticancer treatments on female fertility depends on the women's age at the time of treatment, the chemotherapy protocol, the duration, and total cumulative dose administered.

\footnotetext{
Age

The high susceptibility of the ovaries to chemotherapyinduced toxicity derives from the physiological decline in the number of oocytes from birth to menopause. Approximately, $90 \%$ of oocytes within the female ovary will undergo physiological apoptosis during the fetal or postnatal life (Littley et al. 1989, Johnson et al. 2004). The cellular machinery of the follicles has an inherent high susceptibility to the apoptosis exerted by many chemotherapeutic agents. Chemotherapy-induced ovarian failure is age-dependent, with older age being associated with a greater loss of ovarian reserve. This is due to strong negative correlation between age and the
}

nongrowing follicle pool, representing ovarian reserve (Anderson \& Cameron 2007, Kelsey \& Wallace 2010). Assessing ovarian reserve in young patients may be challenging due to the rise in AMH level before the peak at around age 25 years. Nevertheless, a recent study performed on prepubertal and postpubertal girls treated with chemotherapy indicated that $\mathrm{AMH}$ may be used as a clinically useful marker of damage to the ovarian reserve (Brougham et al. 2012).

\section{Type of treatment}

Anticancer treatments can result in subfertility or infertility due to its impacts on the hypothalamicpituitary-gonadal axis, causing a hypogonadic state in which the ovary is not adequately stimulated, or due to direct gonadotoxic damage to the germ cells. Cranial irradiation >35-40 Gy can impair hypothalamic pituitary function, resulting in hypogonadism through GNRH or FSH/LH deficiency (Gonfloni et al. 2009). Radiation to the pelvis may induce uterine fibrosis, which may complicate future pregnancies. There is no evidence for chemotherapy-induced uterine toxicity (Critchley \& Wallace 2005).

\section{Chemotherapy}

Alkylating agents are overall more toxic to the ovary than other chemotherapy classes, although in preclinical studies, anthracyclines and platinum compounds have also been shown to be toxic to germ cells (Bines et al. 1996, Ben-Aharon et al. 2010). Several clinical studies have demonstrated that chemotherapy-related amenorrhea (CRA) rates varied from 30 to $76 \%$, depending on the average age of the cohort and the chemotherapeutic protocol used (Goodwin et al. 1999, Burstein \& Winer 2000, Stone et al. 2000, Parulekar et al. 2005, Hart 2008, Abusief et al. 2010). CRA occurs in over $90 \%$ of patients treated with high-dose chemotherapies, induction therapies before bone marrow transplantation, or total body irradiation (TBI). Virtually all women undergoing induction chemotherapy and $\mathrm{TBI}$ before transplantation are irreversibly sterilized (Lobo 2005). There is very limited data regarding infertility risk for patients undergoing newer chemotherapy regimens or targeted biological agents.

\section{Mechanisms of chemotherapy-induced ovarian toxicity}

It has been formerly implied that the pathogenesis of chemotherapy-induced ovarian toxicity involves loss of ovarian reserve, and hence several studies have associated it with the mechanism of primary ovarian failure where the loss of ovarian reserve is accelerated (Dnistrian et al. 1983, Dowsett \& Richner 1991). 
A recent study implied that the ovarian response of cancer patients who undergo controlled ovarian hyperstimulation before chemotherapy is diminished even before oncological treatment (Domingo et al. 2012). Several suggested mechanisms that underlie chemotherapy-induced gonadotoxicity have been described and are depicted in Fig. 1.

\section{Direct ovarian toxicity}

Several preclinical studies described the pattern of ovarian impairment, mostly following exposure to alkylating agents. Histological observations revealed apoptosis of primordial follicles (PMF) and primarily apoptosis of pregranulosa cells (Nicosia et al. 1985, Perez et al. 1997, Meirow 1999, 2000, Meirow et al. 1999). The observed reduction in the population of PMF was dose-dependent (Meirow 1999, Meirow et al. 1999). Dividing granulosa cells that populate antral follicles are mostly affected by chemotherapies, while the effect on dormant PMF is variable. The rapid and temporary loss of menses in many patients implies this toxic effect on the mature follicles. Direct effect on oocytes has been observed as some chemotherapies cross the bloodfollicle barrier and reach the oocytes enclosed within the follicles (Perez et al. 1999, Bar-Joseph et al. 2010). Lately, an alternative mechanism has been suggested to explain the variable loss of follicular reserves, the 'burnout' theory on which the destruction of the growing follicles following chemotherapy results in a decrease in cell-derived paracrine growth factors that inhibit PMF recruitment, such as AMH. This may cause an enhanced recruitment of dormant follicles into the pool of actively growing follicles, resulting in a decrease of PMF pool (Meirow et al. 2010).

\section{Ovarian toxicity mediated by an acute vascular insult}

Several studies have documented signs of fibrosis in the cortical stroma, and changes in the capillaries were observed in ovaries exposed to chemotherapy (Marcello et al. 1999, Meirow et al. 2007, Oktem \& Oktay 2007). Using innovative real-time molecular imaging, we observed an acute reduction in ovarian blood flow and disintegration of the vessel wall following in vivo administration of doxorubicin. This phenomenon was doxorubicin-unique and was not evident following exposure to other classes of chemotherapies (Ben-Aharon et al. 2010, Bar-Joseph et al. 2011).

\section{Oxidative stress}

There is limited data from mouse models regarding the initiation of oxidative stress following chemotherapy and irradiation. Thus, it has been shown that antioxidant enzymes may play important roles in follicular survival. Oxidative stress has been associated with cyclophosphamide toxicity in granulosa cells of mature follicles (Chang et al. 1993, Dirven et al. 1994). The active metabolite of cyclophosphamide, 4-hydroxycyclophosphamide (4HC), is formed following oxidation by cytochrome P450 enzymes.

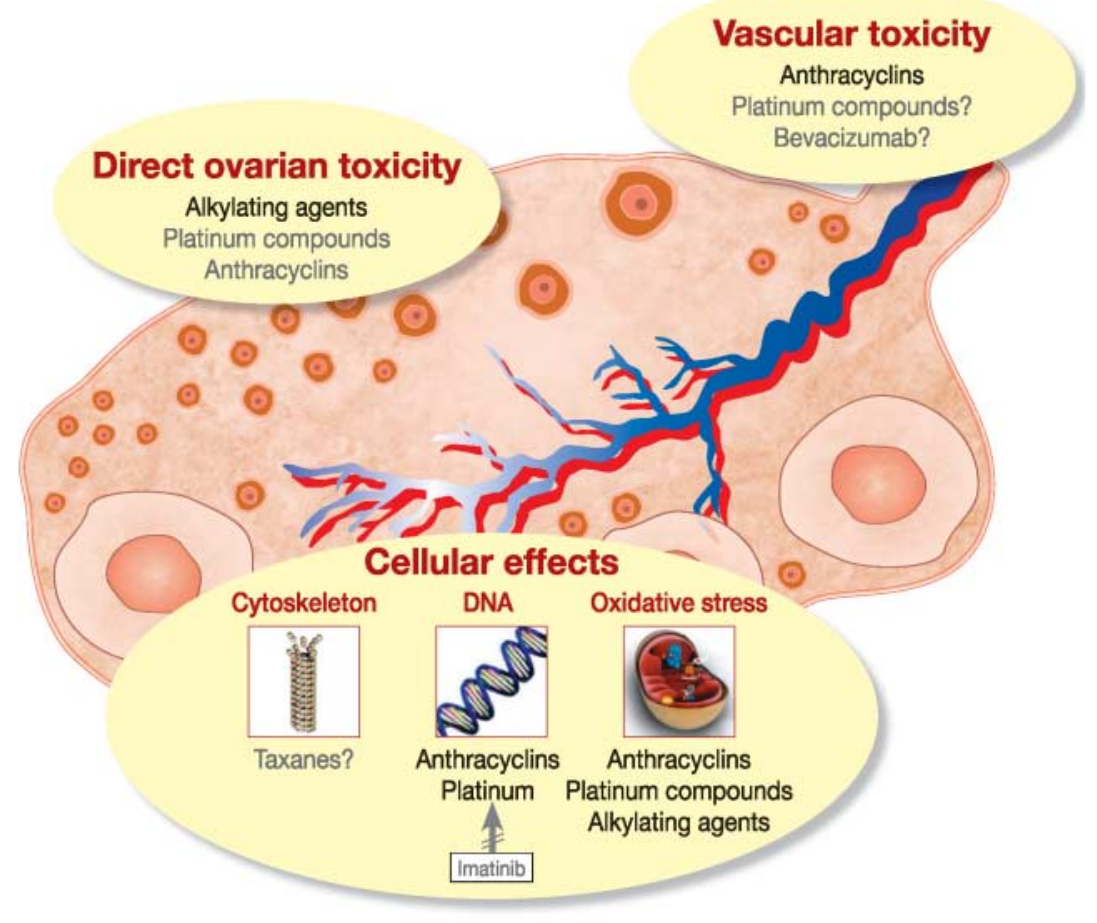

www.reproduction-online.org
Figure 1 Suggested mechanisms for ovarian toxicity. Different classes of chemotherapies exert ovarian toxicity via distinct organ or cellular cascades of events: 1) direct ovarian toxicity or 'ovutoxicity' has been described primarily for alkylating agents that may directly deplete follicular pool. 2) Vascular toxicity may mediate end-organ (i.e. ovarian) damage and has been illustrated in anthracyclinetreated mice by live high-resolution imaging. 3) Direct cellular effects on various components of the ovary have been shown for several classes of chemotherapies that differ on their specific cellular targets. Bold titles represent the well-established evidence for chemotherapy-induced toxicity. 
4HC spontaneously converts into the reactive metabolite phosphoramide mustard. Treatment with cyclophosphamide results in a depletion of glutathione, a crucial cellular antioxidant, and a rise in reactive oxygen species that mediates apoptosis in granulosa cells (Tsai-Turton et al. 2007, Devine et al. 2012).

\section{Gonadotoxicity by class of chemotherapy}

\section{Alkylating agents}

Cyclophosphamide is an alkylating agent that serves as a backbone in many chemotherapeutic regimens and is considered a prototype for gonadotoxic chemotherapy. Cyclophosphamide is not cell cycle-specific, and hence may affect cells that are not actively dividing, such as oocytes or PMF. It has been shown to induce temporary or permanent amenorrhea, and may cause reduced fertility or infertility. Age confers a major determinant for the risk of amenorrhea due to cyclophosphamidecontaining protocols, while rates rise above the age of 35 years and reach $>80 \%$ for women over 40 years old (Parulekar et al. 2005). A recent study provided a long follow up of Hodgkin's lymphoma patients and indicated an increase in risk of premature ovarian failure of $23 \%$ per year of age at treatment (hazard ratio, 1.23 ), corrected with dose of alkylating chemotherapy administered (van der Kaaij et al. 2012).

Destruction of follicles at all stages of development in a dose-dependent manner has been reported in preclinical studies and in human ovarian tissue as well. In mice, a minimal exposure to cyclophosphamide results in a significant annihilation of PMF (Meirow 1999, Meirow et al. 1999). Using a novel xenografting model to characterize the impact of chemotherapy on human PMF reserve, a single injection of cyclophosphamide resulted in a drastic reduction in $\mathrm{PMF}^{\prime}$ density (Reh et al. 2008). This cyclophosphamide-induced toxicity was documented in other studies as well (Jarrell et al. 1987, Plowchalk \& Mattison 1991, Ataya et al. 1995, Meirow 1999, Meirow et al. 1999). In a study that evaluated the reproductive performance and teratogenicity of cyclophosphamide in mice, it has been suggested that the effect of cyclophosphamide on the gametes is influenced by the stage of oocyte maturation at the time of exposure to the agent. Early post-chemotherapy fertilization results in higher rates of pregnancy failure and malformations.

\section{Anthracyclines}

Anthracyclines are widely integrated in a variety of anticancer regimens. Doxorubicin represents a key player of the anthracyclines; it accumulates in both nucleus and mitochondria, initiates oxidative stress, and induces chromosomal obliteration by inhibiting topoisomerase-II. In clinical and cohort studies, there is a wide range of amenorrhea incidence following doxorubicin-based protocols that reflects a tight correlation between the age of the patient and a worsening effect of doxorubicin. While in women between 40 and 49 years of age, doxorubicin-containing regimens were associated with amenorrhea rate of $96 \%$ (Bines et al. 1996), in younger women treated without alkylating chemotherapy, cumulative amenorrhea rates ranged between $<10 \%$ (Brusamolino et al. 2000, Zekri et al. 2008, van der Kaaij et al. 2012) and $\sim 34 \%$ (Cobleigh et al. 1995, Elis et al. 2006). If menstruation returned after treatment, cumulative premature ovarian failure risk was independent of age at treatment.

However, preclinical studies illustrate a pronounced gonadotoxic effect to doxorubicin. It has been shown that doxorubicin induces apoptosis in mammalian oocytes and decreases their survival in vitro significantly (Perez et al. 1997, 1999, Takai etal. 2007, Ting \& Petroff 2010). Most of the studies were performed on ovulated metaphase II (MII) oocytes. We demonstrated that doxorubicin crosses the blood-follicle barrier and directly affects the germinal vesicle (GV) oocytes that represent a more accurate physiological model of oocytes encountering chemotherapy. We observed different sensitivities of GV and MII oocytes to doxorubicin, manifested by different intensity of apoptotic responses, where GV oocytes are more vulnerable. Doxorubicin exerts its toxic effect in oocytes via the mitochondria, concomitant with its known effect on the chromosomes, induction of endoplasmic reticulum (ER)-stress, and a possible increase in intracellular $\mathrm{Ca}^{2+}$, leading to apoptosis, as illustrated in Fig. 2 (Bar-Joseph et al. 2010). This cascade had been formerly introduced in cardiomyocytes (Jang et al. 2004). Our former results indicated an acute doxorubicin-induced ovarian toxicity, manifested by a dramatic reduction in ovulation rate that was partially recovered at later phases and a significant reduction in the population of secondary and PMF 1 month following treatment, as seen in histological sections.

We have established an innovative in vivo real-time molecular imaging platform to track the effect of chemotherapies on the gonads. In vivo magnetic resonance imaging (MRI) depicted a decrease in ovarian size and a marked periovarian edema (Ben-Aharon et al. 2010). We used microscopic ultrasound using microbubbles as a contrast agent to visualize the acute effect of doxorubicin on the ovarian vasculature and revealed a $33 \%$ decrease in ovarian blood volume already $3 \mathrm{~min}$ after doxorubicin injection. Visualization of ovarian microvasculature was obtained by fluorescence optical imaging system, equipped with a confocal fiber microscope. It depicted a pattern of an acute vessels injury, where the wall of the blood vessels became irregular and the fluorescence signal displayed in the small vessels was gradually diminished (Bar-Joseph et al. 2011). Based on other in vitro and in vivo studies that imply the toxicity of doxorubicin to the endothelium, the 


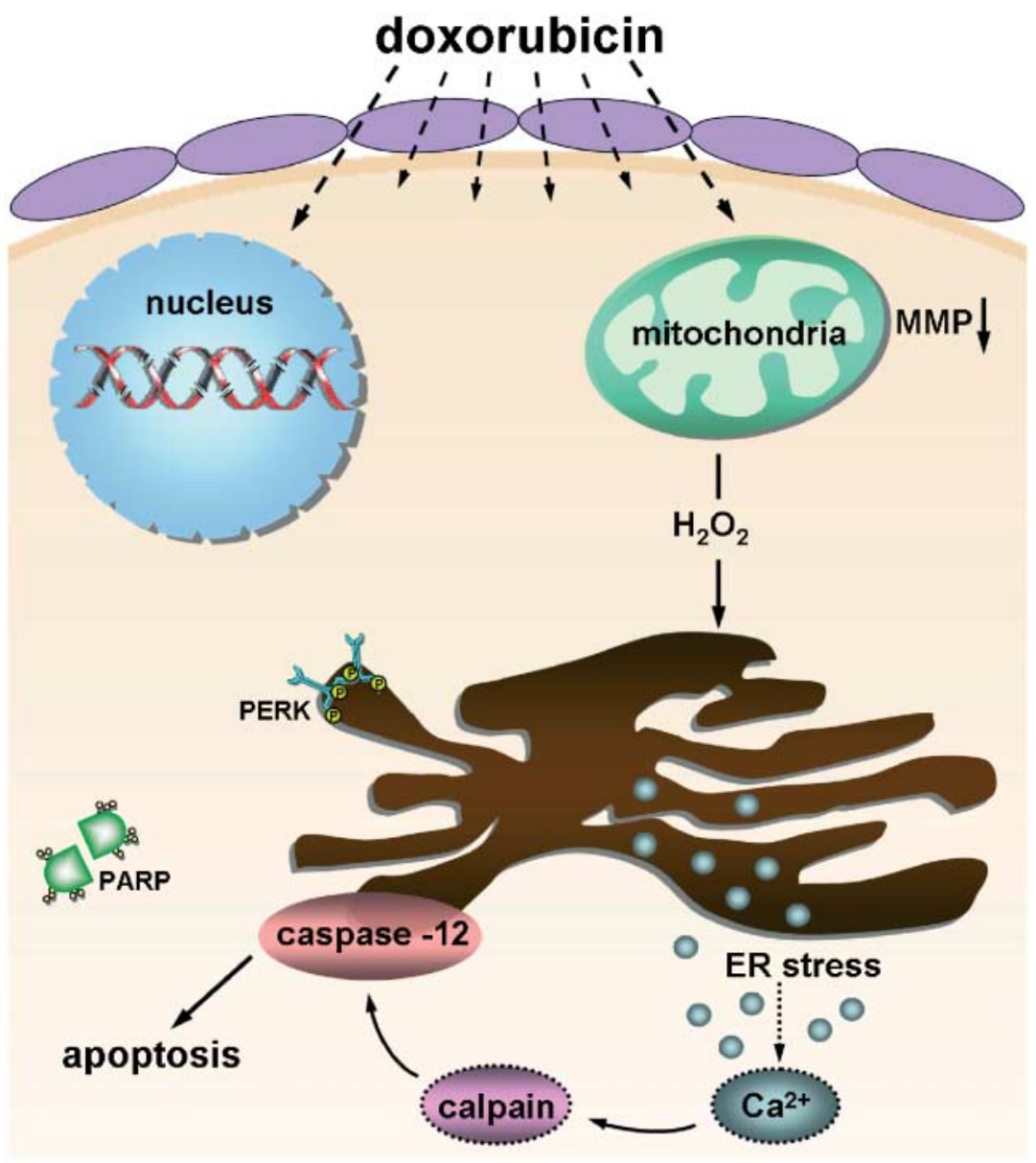

Figure 2 Proposed cellular signal transduction for doxorubicin-induced toxicity within mammalian oocytes. Suggested pathway of doxorubicininduced apoptosis within oocytes involves chromosomal disintegration, activation of the mitochondria, and elevation of superoxides, followed by ER-stress induction. This cascade exerts activation of PERK and caspase-12, a possible increase in intracellular calcium reflected by calpain involvement, and inactivation of PARP. The proposed doxorubicin-induced apoptotic signal transduction observed in oocytes resembles the one studied in cardiomyocytes. acute reduction in gonadal and femoral blood flow and the impairment of the blood vessels wall may mediate the toxic effect doxorubicin exerts on the ovary.

\section{Platinum compounds}

Members of the platinum-based compounds family are a cornerstone of the current antineoplastic treatments in various cancers and are specific inducers of various types of chromosomal damage and DNA cross-links (Blommaert \& Saris 1995). Cisplatin is the prototype and mostly investigated family member. Evidence for platinum-induced ovarian toxicity has been published only with regard to cisplatin. Clinical data is very limited, showing mild to moderate rates of amenorrhea following cisplatin-based treatments (Maneschi et al. 1994, Meirow 2000, Nozaki et al. 2009). The pattern of ovarian injury was studied in an in vitro human tissue model that depicted histological and immunohistochemical changes that accompany PMF destruction: pregranulosa cell swelling with marked accumulation of cytokeratin, pregranulosa cell nuclear swelling, PMF architecture disruption with disappearance of the lumen, and its oocyte (Meirow 2000, Gonfloni et al. 2009). Few studies that appraised cisplatin toxicity in various tissues indicated that the cell cycle phase is a crucial parameter for cisplatin-induced damage, as DNA replication is necessary for the expression of cisplatin effect (Higdon et al. 1992). It has been shown that cisplatin induces a reduction in ovulation rate in rats and a decline in the levels of AMH and inhibin- $\alpha$ (Higdon et al. 1992, Yeh et al. 2006, 2008). It has been implied that cisplatininduced ovarian toxicity may derive from a decline in the level of hyperpolarization-activated cation channels in the ovary. These channels are widely distributed in the ovary and may mediate the effect on oocytes, granulosa cells, and theca cells and contribute to decreased ovarian function (Yeh et al. 2009). The well-documented cisplatin-induced nephrotoxicity also involves differential changes in membrane channels in the kidney (Kishore et al. 2000). Another proposed mechanism for cisplatin-induced ovarian toxicity is that cisplatin leads to the accumulation or activation of p63, a member of the p53 family, and eventually to oocyte death. It was postulated that the DNA damage caused by cisplatin has been shown to activate the c-Abl tyrosine kinase as cisplatin treatment induced a 1.5-fold increase in c-Abl mRNA level, as well as c-Abl nuclear accumulation and cleavage. The putative role of $\mathrm{c}-\mathrm{Abl}$ in the pathogenesis of cisplatin-induced damage rationalized the targeting of 
c-Abl activity with imatinib (see also below), which led to a significant rescue of primary and PMF in ovaries treated simultaneously with cisplatin and imatinib (Gonfloni et al. 2009).

\section{Taxanes}

Taxanes are widely used in various malignancies and have become a pivotal cornerstone in the adjuvant treatment of breast cancer, replacing in specific instances the alkylating-based regimens. Paclitaxel and docetaxel act on the cytoskeleton: they stabilize microtubules and disrupt normal polymerization/depolymerization, leading to an arrest of the cells at the G2-M phase of the cell cycle. The evidence for the potential gonadotoxicity taxanes may confer limited and inconsistent. Few clinical studies have found no additional increase in amenorrhea rates in women treated by taxanecontaining regimens, or a mild increase in a reversible amenorrhea (Davis et al. 2005, Berliere et al. 2008, Reh et al. 2008, Abusief et al. 2010, Pérez-Fidalgo et al. 2010). Nevertheless, several prospective studies have shown that the incidence of amenorrhea in taxanebased chemotherapy regimens was higher than in anthracycline-based chemotherapy regimens (Fornier et al. 2005, Oktay et al. 2005, Han et al. 2009). Women older than 40 years had a greater risk of amenorrhea, with a greater likelihood of irreversibility, than younger women (Petrek et al. 2006, Tham et al. 2007).

In rats, paclitaxel treatment resulted in a decreased number of PMF compared with control (Yucebilgin et al. 2004), indicating that paclitaxel is gonadotoxic. It was also documented that rats exposed to high-dose paclitaxel exhibited loss of fertility, manifested by blocked ovulation, although in a later follow up there was not a significant difference in the number of fetuses, implantation sites, and resorption, suggesting that paclitaxel effect on the ovary may be reversible (Kai et al. 1994). Other studies demonstrated that paclitaxel induces apoptosis mainly in mature follicles and to a lesser extent in a less mature population; accordingly, $E_{2}$ production was affected and decreased serum levels were observed (Tarumi et al. 2009). A clue for the nontoxic effect of paclitaxel on oocytes was provided while treatment with paclitaxel on in vitro matured porcine oocytes had significantly positive effects on morphology, distribution, and ultrastructure of mitochondria and lipid droplets in the oocytes (Fu et al. 2009). In contrary to doxorubicin, the mechanism of potential paclitaxel-induced ovarian toxicity is not mediated by acute vascular injury (Bar-Joseph et al. 2011).

\section{Vinca alkaloids}

No increased risk of ovarian failure was detected in patients treated with plant alkaloids (Meirow 2000,
Zhou et al 2010). Nevertheless, vincristine has been reported as an aneuploidy inducer (Meirow 1999, Meirow et al. 1999).

\section{Antimetabolites}

Fluoropyrimidines are considered as the backbone of adjuvant treatment for colorectal cancer. They act primarily on cells that are actively synthesizing DNA (S-phase of the cell cycle). The widely used agents are 5 -fluorouracil (5-FU) and capecitabine, an oral fluoropyrimidine, which has been shown to confer survival rates similar to 5-FU. The clinical data regarding the impact of fluoropyrimidines on fertility are limited mainly due to the older age of patients and paucity of premenopausal population in the trials. Standard 5-FU-based chemotherapy is considered to have minimal effects on female fertility. There are few reports of premature ovarian failure following 5-FU adjuvant therapy (Azem et al. 2004, Twelves et al. 2005). The antimetabolites methotrexate and 5-FU in the cyclophosphamide, methotrexate, 5-FU (CMF) regimen have not been associated with an increased rate of amenorrhea. While the amenorrhea rate of methotrexate and 5-FU in the adjuvant setting reached $9 \%$, the rate in the standard CMF regimens, using oral cyclophosphamide, was $69 \%$ (Bines et al. 1996).

A study performed on a mouse model shed light on a possible correlation between a potential toxic effect of 5-FU to ovarian function and a circadian timing of administration of the agent. When 5-FU was administered during the estrous phase (immediate postovulatory) of the fertility cycle, female mice suffered a greater subsequent loss of fertility, determined by a decreased successful pregnancy rate, than mice administered with 5-FU during the metestrous, diestrous, or proestrous stages (Hrushesky et al. 1999).

\section{Biological agents}

\section{Imatinib}

Imatinib mesylate is a highly effective, targeted agent used widely to treat Philadelphia-positive leukemia and acts through selective inhibition of tyrosine kinases in other selected cancers. The treatment may last for a long time, offering a prolonged remission. Although mammalian ovaries express several kinases as c-kit, c-abl, and platelet-derived growth factor receptor that are inhibited by imatinib (Hutt et al. 2006), its impact on fertility capacity is not clear. The clinical evidence for a potential gonadotoxic effect is conflicting and is presented as case reports and not as a prospective trial (Hensley \& Ford 2003, Christopoulos et al. 2008, Malozowski et al. 2008, Zamah et al. 2011). Recent study on leukemic mouse model depicted that imatinib mesylate at therapeutic doses showed no effects on either folliculogenesis or 
spermatogenesis, suggesting that the agent does not reduce fertility (Schultheis et al. 2012). Furthermore, it has been suggested that imatinib may exhibit a protective effect on chemotherapy-induced gonadotoxicity. As described earlier, cisplatin rapidly promotes the accumulation of TAp63, a p53 homolog, and ultimately causes cell death. TAp63 expression correlates with oocytes radio sensitivity and is essential for their ensuing DNA damage-induced death within the PMF (Suh et al. 2006). Treatment with imatinib offsets these cisplatininduced effects and further impairs cisplatin-induced depletion of the follicle reserve, suggesting further research of imatinib as a potential protectant of chemotherapy-induced gonadotoxicity (Gonfloni et al. 2009). Nevertheless, imatinib should be avoided before or during pregnancy, due to a higher risk of congenital malformations. The potential impact of other tyrosine kinase inhibitors (TKIs) has not been studied yet; few case reports suggest TKIs do not affect fertility (Conchon et al. 2010, Oweini et al. 2011).

\section{Trastuzumab}

Trastuzumab, a MAB targeting the extracellular domain of the HER2 protein, administered in combination with chemotherapy followed by an extended period of trastuzumab only in the adjuvant setting, improves survival in women with either early or metastatic breast cancer. There is no valid data regarding the impact of trastuzumab on fertility. Unpublished data of a toxicological study conducted in cynomolgus monkeys revealed no evidence of impaired fertility following exposure to high-dose trastuzumab (Genentech 2010: U.S. BL 4851301 Supplement: Trastuzumab Genentech, Inc.). In a clinical study on breast cancer patients, the addition of trastuzumab to chemotherapy did not affect amenorrhea rates (Abusief et al. 2010). Exposure to trastuzumab during pregnancy can result in oligohydramnios and oligohydramnios sequence manifesting as pulmonary hypoplasia, skeletal abnormalities, and neonatal death (Witzel et al. 2008).

\section{AntiVEGF therapy}

Bevacizumab. No published studies have addressed the impact of bevacizumab on fertility. A clinical report of two patients who were treated with bevacizumab due to choroidal melanoma described a transient amenorrhea following bevacizumab treatment (Newman et al. 2011). In 2011, the FDA added a warning label to bevacizumab with regard to ovarian function. Results published by Genentech, the developing company, demonstrate that the incidence of ovarian failure was higher in premenopausal women receiving bevacizumab in combination with adjuvant chemotherapy, compared with those receiving chemotherapy alone for adjuvant treatment for colorectal cancer (34 vs $2 \%$ respectively).
The use of bevacizumab in the adjuvant setting has not been approved (Genetech 2011: U.S. BL 125085 Supplement: Bevacizumab Genentech, Inc.).

\section{Tamoxifen}

Tamoxifen, a selective estrogen receptor modulator, has been widely used in hormone responsive breast cancer. It shares a multifaceted mechanism of tissue-specific activation or inhibition of estrogen signaling. The clinical data with regard to the effect of tamoxifen on fertility are biased with its subsequent use following chemotherapy in most of the studies. Nevertheless, newer technologies of genetic profiling enable the selection of patients with hormone responsive breast cancer that are being treated with endocrine treatment only as a concept of 'tailored' therapy; future studies will therefore reveal the sole effect of tamoxifen on fertility. The pre-clinical data are not consistent: few studies have implied that the addition of tamoxifen to cyclophosphamide resulted in decreased follicle loss and improved reproductive function in rats (Kim et al. 2010, Ting \& Petroff 2010). Another toxicological study on rats that employed a higher dose of tamoxifen showed a disturbance of estrus cycle and a decrease in the number of pregnant rats that were considered to be related to ovarian histopathological changes (Tsujioka et al. 2009). Two clinical studies indicate that tamoxifen may affect the follicular pool, resulting in a delayed fall in $\mathrm{AMH}$ in a group of patients receiving tamoxifen and goserelin. $\mathrm{AMH}$ level continued to further decline, more than the expected physiological decline caused by age alone (Zhou et al. 2010, Anderson \& Cameron 2011). In another follow-up study of menstrual functioning in premenopausal women with invasive breast cancer who received adjuvant chemotherapy with or without tamoxifen, patients receiving tamoxifen therapy were twice as likely to remain amenorrheic compared with those not receiving tamoxifen. However, this association was not statistically significant among women younger than 40 years of age at diagnosis (Abusief et al. 2010). There was no difference in $\mathrm{AMH}$ level between tamoxifen users and no-users, suggesting that only growing follicles were influenced by tamoxifen (Rosendahl et al. 2008). Tamoxifen is contraindicated during pregnancy due to the reports of craniofacial abnormalities associated with tamoxifen use in the first trimester.

\section{Implications for research and practice}

In order to provide young patients with reliable information about the gonadotoxic potential of their anticancer treatment, a better and accurate knowledge regarding the effect of classes of chemotherapy and biological agents is required, as the current data are limited. The available evidence of chemotherapyinduced ovarian toxicity displays several limitations: 
the use of nonuniform definitions of assessing the ovarian reserve, different combinations of drugs in the various studies, small sample size, the heterogeneity of the cohort (mainly wide age range and lack of exclusion of patients with other possible causes of subfertility), and the short follow up of the majority of the studies. Most of the included studies employed resumption of menstrual cycle as an indicator for preserved ovarian function. Numerous studies have demonstrated that menstruating is not an accurate marker and does not reflect reproductive capacity, particularly in women with breast cancer who continue with hormonal therapy (such as tamoxifen) after the cessation of chemotherapy. Other hormonal measurements include: $\mathrm{FSH}$, inhibin- $\beta, \mathrm{AMH}$, and antral follicle count. In recent studies, $\mathrm{AMH}$ shows promise as a reliable hormonal biomarker of reduced ovarian reserve in women who have been previously treated with anticancer treatments. The CCSS represents a well-characterized cohort study that appraises detailed information on reproductive parameters in a large cohort and hence enables to determine the specific long-term effect on fertility, manifested in pregnancies and the occurrence of premature ovarian failure. The fact that childhood cancer survivors who continued to have spontaneous menses more than 5 years after their cancer diagnosis had a risk of developing premature menopause that was 13-fold higher than that in siblings, with a cumulative incidence of $8 \%$ by age 40 years, along with the significant increased use of ART by childhood cancer survivors (Sklar et al. 2006), indicates that the resumption of menses may in some cases be insufficient to predict the future fertility state and that the actual premature ovarian failure rates may be higher than the reported incidence of amenorrhea.

Notably, there is no evidence that the prepubertal ovary is protected from the effects of chemotherapy (Kelsey \& Wallace 2010). However, young patients, due to their larger PMF pool, may have a window of opportunity for fertility before the onset of a premature menopause.

A prospective registry of reproductive outcomes for a long-term follow-up while documenting menstruation, pregnancy rates, and the use of ART along with biomarkers evaluation would enable a better characterization of the actual effect of anticancer treatments on ovarian function. Future preclinical research on specific mechanisms of gonadotoxicity of older and newer drugs would unravel what lies behind gonadotoxicity and may enable the development of novel strategies to preserve fertility.

As the rate of young cancer survivors has grown, fertility issues have become more relevant. Providing cancer patients with timely information related to the potential gonadotoxicity and options for fertility preservation are imperative. Referring the patients to a reproductive specialist may partially alleviate the emotional burden that accompanies the commencement of anticancer treatment and later on facilitates the transition from a cancer patient to a cancer survivor.

\section{Declaration of interest}

The authors declare that there is no conflict of interest that could be perceived as prejudicing the impartiality of the review reported.

\section{Funding}

This review was funded by the Israeli Cancer Association (ICA; research grant no. 20120046).

\section{References}

Abusief ME, Missmer SA, Ginsburg ES, Weeks JC \& Partridge AH 2010 The effects of paclitaxel, dose density, and trastuzumab on treatment-related amenorrhea in premenopausal women with breast cancer. Cancer 116 791-798. (doi:10.1002/cncr.24835)

Anderson RA \& Cameron DA 2007 Assessment of the effect of chemotherapy on ovarian function in women with breast cancer. Journal of Clinical Oncology 25 1630-1631 (author reply 1632). (doi:10.1200/JCO.2006.09.3666)

Anderson RA \& Cameron DA 2011 Pretreatment serum anti-Müllerian hormone predicts long-term ovarian function and bone mass after chemotherapy for early breast cancer. Journal of Clinical Endocrinology and Metabolism 96 1336-1343. (doi:10.1210/jc.2010-2582)

Ataya K, Rao LV, Lawrence E \& Kimmel R 1995 Luteinizing hormonereleasing hormone agonist inhibits cyclophosphamide-induced ovarian follicular depletion on rhesus monkeys. Biology of Reproduction 52 365-372. (doi:10.1095/biolreprod52.2.365)

Azem F, Amit A, Merimsky O \& Lessing JB 2004 Successful transfer of frozen-thawed embryos obtained after subtotal colectomy for colorectal cancer and before fluorouracil-based chemotherapy. Gynecologic Oncology 93 263-265. (doi:10.1016/j.ygyno.2003.12.030)

Bar-Joseph H, Ben-Aharon I, Rizel S, Stemmer SM, Tzabari M \& Shalgi R 2010 Doxorubicin-induced apoptosis in germinal vesicle oocytes. Reproductive Toxicology 30 566-572. (doi:10.1016/j.reprotox.2010. 07.003)

Bar-Joseph H, Ben-Aharon I, Tsarfaty G, Tzabari M, Stemmer SM \& Shalgi R 2011 Novel in vivo imaging of acute chemotherapy-induced vascular toxicity. PLoS One 6 e23492.

Ben-Aharon I, Bar-Joseph H, Tzarfaty G, Kuchinsky L, Rizel S, Stemmer SM \& Shalgi R 2010 Doxorubicin-induced ovarian toxicity. Reproductive Biology and Endocrinology 4 8-20.

Berliere M, Dalenc F, Malingret N, Piette P, Roche H, Donnez J, Symann M, Kerger J \& Machiels JP 2008 Incidence of reversible amenorrhea in women with breast cancer undergoing adjuvant anthracyclinebased chemotherapy with or without docetaxel. BMC Cancer 8 56-64. (doi:10.1186/1471-2407-8-56)

Bines J, Oleske DM \& Cobleigh MA 1996 Ovarian function in premenopausal women treated with adjuvant chemotherapy for breast cancer. Journal of Clinical Oncology 14 1718-1729.

Blommaert FA \& Saris C 1995 Detection of platinum-DNA adducts by ${ }^{32} \mathrm{P}$ postlabelling. Nucleic Acids Research 23 1300-1306. (doi:10.1093/nar/ 23.8.1300)

Brougham MF, Crofton PM, Johnson EJ, Evans N, Anderson RA \& Wallace WH 2012 Anti-Müllerian hormone is a marker of gonadotoxicity in pre- and postpubertal girls treated for cancer: a prospective study. Journal of Clinical Endocrinology and Metabolism 97 2059-2067. (doi:10.1210/jc.2011-3180)

Brusamolino E, Lunghi F, Orlandi E, Astori C, Passamonti F, Baraté C, Pagnucco G, Baio A, Franchini P, Lazzarino M et al. 2000 Treatment of early-stage Hodgkin's disease with four cycles of ABVD followed by adjuvant radio-therapy: analysis of efficacy and long-term toxicity. Haematologia 85 1032-1039. 
Burstein HJ \& Winer EP 2000 Primary care for survivors of breast cancer. New England Journal of Medicine 343 1086-1894. (doi:10.1056/ NEJM200010123431506)

Chang TK, Weber GF, Crespi CL \& Waxman DJ 1993 Differential activation of cyclophosphamide and ifosphamide by cytochromes P-450 2B and 3A in human liver microsomes. Cancer Research 53 5629-5637.

Christopoulos C, Dimakopoulou V \& Rotas E 2008 Primary ovarian insufficiency associated with imatinib therapy. New England Journal of Medicine 358 1079-1080. (doi:10.1056/NEJMc0707841)

Cobleigh MA, Bines J, Harris D, La Follette S, Lincoln ST \& Wolter JM 1995 Amenorrhea following adjuvant chemotherapy for breast cancer. Proceedings of the American Society of Clinical Oncology 14158 (abstract).

Conchon M, Sanabani SS, Serpa M, Novaes MM, Nardinelli L, Ferreira PB, Dorliac-Llacer PE \& Bendit I 2010 Successful pregnancy and delivery in a patient with chronic myeloid leukemia while on dasatinib therapy. Advances in Hematology. (doi:10.1155/2010/136252)

Critchley HO \& Wallace WH 2005 Impact of cancer treatment on uterine function. Journal of the National Cancer Institute. Monographs 34 64-68. (doi:10.1093/jncimonographs/lgi022)

Davis AL, Litus M \& Mintzer DM 2005 Chemotherapy-induced amenorrhea from adjuvant breast cancer treatment: the effect of the addition of taxanes. Clinical Breast Cancer 6 421-424. (doi:10.3816/CBC.2005.n. 046)

Devine PJ, Perreault SD \& Luderer U 2012 Roles of reactive oxygen species and antioxidants in ovarian toxicity. Biology of Reproduction 8627. (doi:10.1095/biolreprod.111.095224)

Dirven HA, van Ommen B \& van Bladeren PJ 1994 Involvement of human glutathione S-transferase isoenzymes in the conjugation of cyclophosphamide metabolites with glutathione. Cancer Research 54 6215-6220.

Dnistrian AM, Schwartz MK, Fracchia AA, Kaufman FJ, Hakes TB \& Currie VE 1983 Endocrine consequences of CMF adjuvant therapy in premenopausal and postmenopausal breast cancer patients. Cancer 51 803-807. (doi:10.1002/1097-0142(19830301)51:5<803::AID-CNCR 2820510509>3.0.CO;2-V)

Domingo J, Guillén V, Ayllón Y, Martínez M, Muñoz E, Pellicer A \& Garcia-Velasco JA 2012 Ovarian response to controlled ovarian hyperstimulation in cancer patients is diminished even before oncological treatment. Fertility and Sterility 97 930-934. (doi:10.1016/j.fertnstert. 2012.01.093)

Dowsett M \& Richner J 1991 Effects of cytotoxic chemotherapy on ovarian and adrenal steroidogenesis in pre-menopausal breast cancer patients. Oncology 48 215-220. (doi:10.1159/000226930)

Elis A, Tevet A, Yerushalmi R, Blickstein D, Bairy O, Dann EJ, Blumenfeld Z, Abraham A, Manor Y, Shpilberg O et al. 2006 Fertility status among women treated for aggressive non-Hodgkin's lymphoma. Leukemia \& Lymphoma 47 623-627. (doi:10.1080/10428190500353877)

Fornier MN, Modi S, Panageas KS, Norton L \& Hudis C 2005 Incidence of chemotherapy- induced, long-term amenorrhea in patients with breast carcinoma age 40 years and younger after adjuvant anthracycline and taxane. Cancer 104 1575-1579. (doi:10.1002/cncr.21385)

Fu XW, Shi WQ, Zhang QJ, Zhao XM, Yan CL, Hou YP, Zhou GB, Fan ZQ, Suo L, Wusiman A et al. 2009 Positive effects of taxol pretreatment on morphology, distribution and ultrastructure of mitochondria and lipid droplets in vitrification of in vitro matured porcine oocytes. Animal Reproduction Science 115 158-168. (doi:10.1016/j.anireprosci.2008. 12.002)

Gonfloni S, Di Tella L, Caldarola S, Cannata SM, Klinger FG, Di Bartolomeo C, Mattei M, Candi E, De Felici M, Melino G et al. 2009 Inhibition of the c-Abl-TAp63 pathway protects mouse oocytes from chemotherapyinduced death. Nature Medicine 15 1179-1185. (doi:10.1038/nm.2033)

Goodwin PJ, Ennis M, Pritchard KI, Trudeau M \& Hood N 1999 Risk of menopause during the first year after breast cancer diagnosis. Journal of Clinical Oncology 17 2365-2370.

Green DM, Kawashima T, Stovall M, Leisenring W, Sklar CA, Mertens AC, Donaldson SS, Byrne J \& Robison LL 2010 Fertility of male survivors of childhood cancer: a report from the Childhood Cancer Survivor Study. Journal of Clinical Oncology 28 332-339. (doi:10.1200/JCO.2009.24. 9037)

Han HS, Ro J, Lee KS, Nam BH, Seo JA, Lee DH, Lee H, Lee ES, Kang HS \& Kim SW 2009 Analysis of chemotherapy-induced amenorrhea rates by three different anthracycline and taxane containing regimens for early breast cancer. Breast Cancer Research and Treatment 115 335-342. (doi:10.1007/s10549-008-0071-9)

Hart R 2008 Preservation of fertility in adults and children diagnosed with cancer. BMJ 337 a2045. (doi:10.1136/bmj.a2045)

Higdon RE, Marchetti F, Mailhes JB \& Phillips GL 1992 The effects of cisplatin on murine metaphase II oocytes. Gynecologic Oncology 147 348-352. (doi:10.1016/0090-8258(92)90138-9)

Hensley ML \& Ford JM 2003 Imatinib treatment: specific issues related to safety, fertility, and pregnancy. Seminars in Hematology 40 (Suppl 2) 21-25. (doi:10.1053/shem.2003.50038)

Hrushesky WJ, Vyzula R \& Wood PA 1999 Fertility maintenance and 5 -fluorouracil timing within the mammalian fertility cycle. Reproductive Toxicology 13 413-420. (doi:10.1016/S0890-6238(99)00037-4)

Hutt KJ, McLaughlin EA \& Holland MK 2006 Kit ligand and C-Kit have diverse roles during mammalian oogenesis and folliculogenesis. Molecular Human Reproduction 12 61-69. (doi:10.1093/molehr/ gal010)

Jang YM, Kendaiah S, Drew B, Phillips T, Selman C, Julian D \& Leeuwenburgh C 2004 Doxorubicin treatment in vivo activates caspase-12 mediated cardiac apoptosis in both male and female rats. FEBS Letters 577 483-490. (doi:10.1016/j.febslet.2004.10.053)

Jarrell J, Young Lai EV, Barr R, McMahon A, Belbeck L \& O'Connell G 1987 Ovarian toxicity of cyclophosphamide alone and in combination with ovarian irradiation in the rat. Cancer Research 47 2340-2343.

Jemal A, Siegel R, Ward E, Hao Y, Xu J, Murray T \& Thun MJ 2008 Cancer statistics, 2008. CA: A Cancer Journal for Clinicians 58 71-96. (doi:10. 3322/CA.2007.0010)

Johnson J, Canning J, Kaneko T, Pru JK \& Tilly JL 2004 Germline stem cells and follicular renewal in the postnatal mammalian ovary. Nature $\mathbf{2 8}$ 145-150. (doi:10.1038/nature02316)

van der Kaaij MA, Heutte N, Meijnders P, Abeilard-Lemoisson E, Spina $M$, Moser EC, Allgeier A, Meulemans B, Simons AH, Lugtenburg PJ et al. 2012 Premature ovarian failure and fertility in long-term survivors of Hodgkin's lymphoma: a European Organisation for Research and Treatment of Cancer Lymphoma Group and Groupe d'Etude des Lymphomes de I'Adulte Cohort Study. Journal of Clinical Oncology 30 291-299. (doi:10.1200/JCO.2011.37.1989)

Kai S, Kohmura H, Hiraiwa E, Koizumi S, Ishikawa K, Kawano S, Kuroyanagi K, Hattori N, Chikazawa H \& Kondoh H 1994 Reproductive and developmental toxicity studies of paclitaxel. (I) Intravenous administration to rats prior to and in the early stages of pregnancy. Journal of Toxicological Sciences 19 57-67. (doi:10.2131/jts.19. Supplementl_57)

Kelsey TW \& Wallace WH 2010 Human ovarian reserve from conception to menopause. PLoS ONE 5 e8772. (doi:10.1371/journal.pone.0008772)

Kim SS, Lee JR, Jee BC, Suh CS, Kim SH, Ting A \& Petroff B 2010 Use of hormonal protection for chemotherapy-induced gonadotoxicity. Clinical Obstetrics and Gynecology 53 740-752. (doi:10.1097/GRF. Ob013e3181f96cb1)

Kishore BK, Krane CM, Di Iulio D, Menon AG \& Cacini W 2000 Expression of renal aquaporins 1,2, and 3 in a rat model of cisplatin-induced polyuria. Kidney International 58 701-711. (doi:10.1046/j.1523-1755. 2000.00216.x)

Littley MD, Shalet SM \& Beardwell CG 1989 Hypopituitarism following external radiotherapy for pituitary tumours in adults. Quarterly Journal of Medicine 70 145-160.

Lobo RA 2005 Potential options for preservation of fertility in women. New England Journal of Medicine 353 64-73. (doi:10.1056/NEJMra043475)

Madanat LM, Malila N, Dyba T, Hakulinen T, Sankila R, Boice JD Jr \& Lähteenmäki PM 2008 Probability of parenthood after early onset cancer: a population-based study. International Journal of Cancer 123 2891-2898. (doi:10.1002/ijc.23842)

Magelssen H, Melve KK, Skjaerven R \& Fosså SD 2008 Parenthood probability and pregnancy outcome in patients with a cancer diagnosis during adolescence and young adulthood. Human Reproduction 23 178-186. (doi:10.1093/humrep/dem362)

Malozowski S, Nelson L \& Calis KA 2008 More on ovarian insufficiency with imatinib. New England Journal of Medicine 358 2648-2649. (doi:10.1056/NEJMc080707) 
Maneschi F, Benedetti-Panici P, Scambia G, Salerno MG, D'Agostino G \& Mancuso S 1994 Menstrual and hormone patterns in women treated with high-dose cisplatin and bleomycin. Gynecologic Oncology 54 345-348. (doi:10.1006/gyno.1994.1221)

Marcello MF, Nuciforo G, Romeo R, Dino GD, Russo I, Russo A, Palumbo G \& Schilirò G 1999 Structural and ultrastructural study of the ovary in childhood leukemia after successful treatment. Cancer $\mathbf{6 6}$ 2099-2104. (doi:10.1002/1097-0142(19901115)66:10<2099::AID-CN CR2820661010>3.0.CO;2-3)

Meirow D 1999 Ovarian injury and modern options to preserve fertility in female cancer patients treated with high dose radiochemotherapy for hemato-oncological neoplasias and other cancers. Leukemia \& Lymphoma 33 65-76.

Meirow D 2000 Reproduction post-chemotherapy in young cancer patients. Molecular and Cellular Endocrinology 169 123-131. (doi:10.1016/ S0303-7207(00)00365-8)

Meirow D, Nugent D, Epstein M, Livni N \& Gosden RG 1998 An in vitro study of the effects of chemotherapy on human primordial follicles. Eur. Soc. Hum Reprod (ESHRE meeting), Goteburg, Sweden.

Meirow D, Lewis H, Nugent D \& Epstein M 1999 Subclinical depletion of primordial follicular reserve in mice treated with cyclophosphamide: clinical importance and proposed accurate investigative tool. Human Reproduction 14 1903-1907. (doi:10.1093/humrep/14.7.1903)

Meirow D, Dor J, Kaufman B, Shrim A, Rabinovici J, Schiff E, Raanani H, Levron J \& Fridman E 2007 Cortical fibrosis and blood-vessels damage in human ovaries exposed to chemotherapy chemotherapy. Potential mechanisms of ovarian injury. Human Reproduction 22 1626-1633. (doi:10.1093/humrep/dem027)

Meirow D, Biederman H, Anderson RA \& Wallace WH 2010 Toxicity of chemotherapy and radiation on female reproduction. Clinical Obstetrics and Gynecology 53 727-739. (doi:10.1097/GRF.0b013e 3181f96b54)

Newman H, Finger PT, Chin KJ \& Pavlick AC 2011 Systemic bevacizumab (avastin) for exudative retinal detachment secondary to choroidal melanoma. European Journal of Ophthalmology 21 796-801. (doi:10.5301/EJO.2011. 6477)

Nicosia SV, Matus-Ridley M \& Meadows AT 1985 Gonadal effects of cancer therapy in girls. Cancer 55 2364-2372. (doi:10.1002/1097-0142 (19850515)55:10<2364::AID-CNCR2820551011>3.0.CO;2-E)

Nozaki Y, Furubo E, Matsuno T, Fukui R, Kizawa K, Kozaki T \& Sanzen T 2009 Collaborative work on evaluation of ovarian toxicity. 6) Two- or four-week repeated-dose studies and fertility study of cisplatin in female rats. Journal of Toxicological Sciences 34 (Suppl 1) SP73-SP81. (doi:10.2131/jts.34.S73)

Oktay K, Libertella B \& Oktem O 2005 The impact of paclitaxel on menstrual function. Breast Cancer Research and Treatment 94 $271 \mathrm{~s}-272 \mathrm{~s}$.

Oktem O \& Oktay K 2007 Quantitative assessment of the impact of chemotherapy on ovarian follicle reserve and stromal function. Cancer 110 2222-2229. (doi:10.1002/cncr.23071)

Oweini H, Otrock ZK, Mahfouz RA \& Bazarbachi A 2011 Successful pregnancy involving a man with chronic myeloid leukemia on dasatinib. Archives of Gynecology and Obstetrics 283 133-134. (doi:10.1007/ s00404-010-1501-6)

Parulekar WR, Day AG, Ottaway JA, Shepherd LE, Trudeau ME, Bramwell V, Levine M, Pritchard KI \& National Cancer Institute of Canada Clinical Trials Group 2005 Incidence and prognostic impact of amenorrhea during adjuvant therapy in high-risk premenopausal breast cancer: analysis of a National Cancer Institute of Canada Clinical Trials Group Study - NCIC CTG MA.5. Journal of Clinical Oncology 23 6002-6008. (doi:10.1200/JCO.2005.07.096)

Perez GI, Knudson CM, Leykin L, Korsmeyer SJ \& Tilly JL 1997 Apoptosisassociated signaling pathways are required for chemotherapy-mediated female germ cell destruction. Nature Medicine 3 1228-1232. (doi:10. 1038/nm1197-1228)

Perez GI, Tao XJ \& Tilly JL 1999 Fragmentation and death (a.k.a. apoptosis) of ovulated oocytes. Molecular Human Reproduction 5 414-420. (doi:10.1093/molehr/5.5.414)

Perez GI, Maravei DV, Trbovich AM, Cidlowski JA, Tilly JL \& Hughes FM Jr 2000 Identification of potassium-dependent and -independent components of the apoptotic machinery in mouse ovarian germ cells and granulosa cells. Biology of Reproduction 63 1358-1369. (doi:10. 1095/biolreprod63.5.1358)

Pérez-Fidalgo JA, Roselló S, García-Garré E, Jordá E, Martín-Martorell P, Bermejo B, Chirivella I, Guzman C \& Lluch A 2010 Incidence of chemotherapy-induced amenorrhea in hormone-sensitive breast cancer patients: the impact of addition of taxanes to anthracyclinebased regimens. Breast Cancer Research and Treatment 120 245-251. (doi:10.1007/s10549-009-0426-x)

Petrek JA, Naughton MJ, Case LD, Paskett ED, Naftalis EZ, Singletary SE \& Sukumvanich P 2006 Incidence, time course, and determinants of menstrual bleeding after breast cancer treatment: a prospective study. Journal of Clinical Oncology 24 1045-1051. (doi:10.1200/JCO.2005.03. 3969)

Plowchalk DR \& Mattison DR 1991 Phosphoramide mustard is responsible for the ovarian toxicity of cyclophosphamide. Toxicology and Applied Pharmacology 107 472-481. (doi:10.1016/0041-008X(91)90310-B)

Reh A, Oktem O \& Oktay K 2008 Impact of breast cancer chemotherapy on ovarian reserve: a prospective observational analysis by menstrual history and ovarian reserve markers. Fertility and Sterility 90 1635-1639. (doi:10.1016/j.fertnstert.2007.09.048)

Rosendahl M, Andersen CY, Ernst E, Westergaard LG, Rasmussen PE, Loft A \& Andersen AN 2008 Ovarian function after removal of an entire ovary for cryopreservation of pieces of cortex prior to gonadotoxic treatment: a follow-up study. Human Reproduction 23 2475-2483. (doi:10.1093/ humrep/den248)

Schover LR 2008 Premature ovarian failure and its consequences: vasomotor symptoms, sexuality, and fertility. Journal of Clinical Oncology 26 753-758. (doi:10.1200/JCO.2007.14.1655)

Schultheis B, Nijmeijer BA, Yin H, Gosden RG \& Melo JV 2012 Imatinib mesylate at therapeutic doses has no impact on folliculogenesis or spermatogenesis in a leukaemic mouse model. Leukemia Research $\mathbf{3 6}$ 271-274. (doi:10.1016/j.leukres.2011.09.025)

Sklar CA, Mertens AC, Mitby P, Whitton J, Stovall M, Kasper C, Mulder J, Green D, Nicholson HS, Yasui Y et al. 2006 Premature menopause in survivors of childhood cancer: a report from the childhood cancer survivor study. Journal of the National Cancer Institute 98 890-896.

Stone ER, Slack RS \& Novielli A 2000 Rate of chemotherapy related amenorrhea (CRA) associated with adjuvant adriamycin and cytoxan (AC) and adriamycin and cytoxan followed by taxol $(\mathrm{AC}+\mathrm{T})$ in early stage breast cancer [abstract]. Breast Cancer Research and Treatment 64 61 (Abstract 224).

Suh EK, Yang A, Kettenbach A, Bamberger C, Michaelis AH, Zhu Z, Elvin JA, Bronson RT, Crum CP \& McKeon F 2006 p63 protects the female germ line during meiotic arrest. Nature 444 624-628. (doi:10. 1038/nature05337)

Takai Y, Matikainen T, Jurisicova A, Kim MR, Trbovich AM, Fujita E, Kim MR, Trbovich AM, Fujita E, Nakagawa T et al. 2007 Caspase-12 compensates for lack of caspase-2 and caspase- 3 in female germ cells. Apoptosis 12 791-800. (doi:10.1007/s10495-006-0022-z)

Tarumi W, Suzuki N, Takahashi N, Kobayashi Y, Kiguchi K, Sato K \& Ishizuka B 2009 Ovarian toxicity of paclitaxel and effect on fertility in the rat. Journal of Obstetrics and Gynaecology Research 3 414-420. (doi:10.1111/j.1447-0756.2009.01023.x)

Tham YL, Sexton K, Weiss H, Elledge R, Friedman LC \& Kramer R 2007 The rates chemotherapy-induced amenorrhea in patients treated with adjuvant doxorubicin and cyclophosphamide followed by a taxane. American Journal of Clinical Oncology 30 126-132. (doi:10.1097/01. coc.0000251398.57630.4f)

Ting AY \& Petroff BK 2010 Tamoxifen decreases ovarian follicular loss from experimental toxicant DMBA and chemotherapy agents cyclophosphamide and doxorubicin in the rat. Journal of Assisted Reproduction and Genetics 27 591-597. (doi:10.1007/s10815-010-9463-y)

Tsai-Turton M, Luong BT, Tan Y \& Luderer U 2007 Cyclophosphamide induced apoptosis in COV434 human granulosa cells involves oxidative stress and glutathione depletion. Toxicological Sciences 98 216-230. (doi:10.1093/toxsci/kfm087)

Tsujioka S, Ban Y, Wise LD, Tsuchiya T, Sato T, Matsue K, Ikeda T, Sasaki M \& Nishikibe M 2009 Collaborative work on evaluation of ovarian toxicity. 3) Effects of 2- or 4- week repeated-dose toxicity and fertility studies with tamoxifen in female rats. Journal of Toxicological Sciences 34 (Suppl 1) SP43-SP51. (doi:10.2131/jts.34.S43) 
Twelves C, Wong A, Nowacki MP, Abt M, Burris H Jr, Carrato A, Cassidy J, Cervantes A, Fagerberg J, Georgoulias V et al. 2005 Capecitabine as adjuvant treatment for stage III colon cancer. New England Journal of Medicine 352 2696-2704. (doi:10.1056/NEJMoa043116)

Witzel ID, Müller V, Harps E, Janicke F \& Dewit M 2008 Trastuzumab in pregnancy associated with poor fetal outcome. Annals of Oncology 19 191-192. (doi:10.1093/annonc/mdm542)

Yeh J, Kim B, Liang YJ \& Peresie J 2006 Müllerian inhibiting substance as a novel biomarker of cisplatin-induced ovarian damage. Biochemical and Biophysical Research Communications 348 337-344. (doi:10.1016/j. bbrc.2006.06.195)

Yeh J, Kim BS, Liang YJ \& Peresie J 2008 Baseline and stimulated serum inhibin levels as biomarkers of cisplatin-induced ovarian damage in female rats. American Journal of Obstetrics and Gynecology 198 e1-e6.

Yeh J, Kim BS, Peresie J \& Page C 2009 Declines in levels of hyperpolarization-activated cation $(\mathrm{HCN})$ channels in the rat ovary after cisplatin exposure. Reproductive Sciences 16 986-994. (doi:10. 1177/1933719109339217)

Yucebilgin MS, Terek MC, Ozsaran A, Akercan F, Zekioglu O, Isik E \& Erhan Y 2004 Effect of chemotherapy on primordial follicular reserve of rat: an animal model of premature ovarian failure and infertility. Australian \& New Zealand Journal of Obstetrics \& Gynaecology $4 \mathbf{4}$ 6-9. (doi:10.1111/j.1479-828X.2004.00143.x)

Zamah AM, Mauro MJ, Druker BJ, Oktay K, Egorin MJ, Cedars MI \& Rosen MP 2011 Will imatinib compromise reproductive capacity? Oncologist 16 1422-1427. (doi:10.1634/theoncologist.2011-0137)

Zekri JM, El-Helw LM, Purohit OP, Hatton MQ \& Coleman RE 2008 Epirubicin/vinorelbine adjuvant chemotherapy in young women with breast cancer is associated with preservation of menstrual function. Clinical Oncology 20 513-516. (doi:10.1016/j.clon.2008.03.016)

Zhou WB, Yin H, Liu XA, Zha XM, Chen L, Dai JC, Tao AD, Chen L, Ma JJ, Ling LJ et al. 2010 Incidence of chemotherapy-induced amenorrhea associated with epirubicin, docetaxel and navelbine in younger breast cancer patients. BMC Cancer 10 281. (doi:10.1186/1471-2407-10-281)

Received 3 April 2012

First decision 25 May 2012

Accepted 30 May 2012 